\title{
Design and Testing of ECE Radiometer for Sinp-Tokamak
}

\author{
R. Ray, \\ Saha Institute of Nuclear Physics, Calcutta, India \\ C. A. J. Hugenholtz, and M. J. van de Pol \\ FOM-Institute for plasma physics 'Rijnhuizen', \\ P.O. Box 1207.3430 BE, Nieuwegein, The Netherlands
}

Received on 26 June, 2001

\begin{abstract}
A multi channel ECE Radiometer, in the frequency range of 75 - $110 \mathrm{GHz}$, has been designed, constructed and tested for the SINP-Tokamak to diagnose second harmonic X-mode electron cyclotron emission (ECE). This frequency range covers approximately $80 \%$ of the plasma cross section in case of a magnetic field of $\mathrm{B}=1.5 \mathrm{~T}$. For the maximum field of the SINP-Tokamak B $=2.0 \mathrm{~T}$, about $50 \%$ is covered. The ECE radiation is received by a single horn antenna with a gain of approximately 30 $\mathrm{dB}$. To increase the spatial resolution perpendicular to the line of sight, a focusing lens is employed. The incoming radiation power is split into two branches by a $3 \mathrm{~dB}$ coupler. Before down conversion of the input spectrum, the upper and the lower side band are selected respectively by means of a high pass and a low pass filter. Behind the filters, identical components are employed. The local oscillator frequency is $92 \mathrm{GHz}$ for both mixers. The IF frequency range after down conversion is 2 to $18 \mathrm{GHz}$. The IF spectrum is amplified by two amplifiers with a total gain of approximately $60 \mathrm{~dB}$. The outputs of these amplifiers are split into 6 branches. The frequency selection is made by band pass filters and the received power is detected by Schottky diode detectors followed by video amplification and filtering. The data is recorded by an ADC with $1 \mathrm{MHz}$ sampling rate. The radiometer has been tested and calibrated with a high temperature blackbody radiator. The effective input noise temperature for the radiometer channels is found to be in the order of 0.1 $\mathrm{eV}$. The linear regime of operation extends up to an input power which corresponds to an electron temperature of approximately $200 \mathrm{eV}$. Above this temperature the $1 \mathrm{~dB}$ compression point of the Schottky diode detectors is exceeded and the IF gain should be reduced. This can be easily done by incorporating appropriate attenuator behind the IF amplifiers.
\end{abstract}

\section{Introduction}

A radiometer using the technique of heterodyne for measuring microwaves was first reported in 1946 [1]. This technique is used for spectral decomposition of the radiation received by the radiometer. A heterodyne radiometer has several advantages over the other types of receivers. Some of these advantages are high selectivity, high stability, high accuracy and very good signal to noise ratio. Recent developments in microwave technology have been intelligently exploited to measure high frequency up to $170 \mathrm{GHz}$ with much better frequency resolution and signal to noise ratio [2,3]. A spatial resolution of $\Delta R / R \leq 1 \%$, a temporal resolution of $\geq 100$ $\mathrm{kHz}$ and a temperature resolution of $\Delta T / T \leq 1 \%$ are achievable with radiometer [4].

The principles of heterodyne detection has three dis- tinct processes involved in it. First, the radio frequency (RF) signal which is received by the radiometer is mixed with the signal from a local oscillator. In this stage the frequency spectrum of the original signal is translated to a much lower frequency named as intermediate frequency (IF). The resulting intermediate frequency signal is subsequently amplified and finally detected and processed in a video circuit. In the process of frequency down-conversion three essential components are identified. These are local oscillator, mixer and an intermediate frequency output port. If the RF frequency is $f_{r f}$ and the local oscillator signal has a frequency of $f_{l o}$ respectively then the mixer produces signals of frequencies $f_{i f}=\left|f_{r f} \pm f_{l o}\right|$ and also of many combinations of the respective harmonics. Only the difference frequency $\left|f_{r f}-f_{l o}\right|$ is of interest. Output signals with high frequency are not desirable and can be filtered out 
by appropriate filtering circuits.

The super heterodyne radiometer can be utilized to obtain the spatial and temporal profiles of electron temperature in a magnetically confined plasma through a measurement of the electron cyclotron emission (ECE) [5-8]. The gyration of electrons around the magnetic field lines gives rise to emission (in the non relativistic case) at gyro frequency

$$
\omega_{c e}=\frac{e B}{m_{e} c}
$$

and its harmonics. In a tokamak, the largest component of the magnetic field, the toroidal magnetic field, $B_{T}$ varies inversely with the major radius $R$. In the weakly relativistic regime, the emission in a direction to the perpendicular to the magnetic field is broadened due to the magnetic field inhomogeneity. The frequency of emission is therefore directly related to the spatial position. The temperature profile $T(R)$ can therefore be obtained by a measurement of the emission spectrum from the tokamak plasma.

For a plasma in thermodynamic equilibrium, the specific intensity of emitted radiation is given by

$$
I(\omega)=I_{B}(\omega)\left(1-e^{-\tau}\right)
$$

where $I_{B}(\omega)=\omega^{2} k_{B} T_{e} / 8 \pi^{3} c^{2}$ is the blackbody emission, where $I(\omega)$ is intensity of radiation emitted by plasma, $T_{e}$ is plasma temperature, $k_{B}$ is Boltzmann's constant and $\tau$ is optical thickness.

Re-writing we get

$$
T_{e}=\frac{8 \pi^{3} c^{2} I(\omega)}{\omega^{2} k_{B}\left(1-e^{-\tau}\right)}
$$

For an optically thick plasma $(\tau>>1)$ there is a reabsorption of emitted radiation and $I(\omega)=I_{B}(\omega)$, so that the observed intensity is proportional to the temperature [5]. For $\tau<<1$ plasma is optically thin and there is negligible reabsorption of the radiation emitted.

\section{The sinp radiometer}

The SINP tokamak parameters are $R_{0}=30 \mathrm{~cm}, a=$ $7.5 \mathrm{~cm}$, maximum value of toroidal magnetic field $=2$ $\mathrm{T}$; peak electron density $=3 \times 10^{19} \mathrm{~m}^{-3}$ and peak electron temperature $=300 \mathrm{eV}$. [9]. In the equatorial plane, the plasma radius varies from $22.5 \mathrm{~cm}$ to $37.5 \mathrm{~cm}$. For a toroidal magnetic field $\mathrm{B}=1.3 \mathrm{~T}$ at the centre, the second harmonic of cyclotron frequency varies from 58 $\mathrm{GHz}$ to $97 \mathrm{GHz}$.

The optical depth for the second harmonic extraordinary wave has been calculated and has been found to be greater than 1 for the above working conditions. The following radial profiles of density, toroidal magnetic field and temperature have been utilized to calculate the profile dependence of the right hand cut off frequency $f_{R}$ and the upper hybrid frequency $f_{U H}$ [10].

$$
\begin{gathered}
n_{e}=n_{e 0}\left(1-r^{2} / a^{2}\right), \\
B_{T}=B_{0} /\left(1+r / R_{0}\right), \\
T_{e}=T_{e 0}\left(1-r^{2} / a^{2}\right) \\
f_{R}=\frac{f_{c e}}{2}+\left(\frac{f_{c e}^{2}}{4}+f_{p e}^{2}\right)^{1 / 2}, \\
f_{U H}=\left(f_{p e}^{2}+f_{c e}^{2}\right)^{1 / 2}
\end{gathered}
$$

with $f_{c e}=\omega_{c e} / 2 \pi$.

The accessibility calculations reveal that the second harmonic extraordinary mode can escape the plasma for values of density $\approx 3 \times 10^{19} \mathrm{~m}^{-3}$.

A twelve channel ECE Radiometer (Fig. 1), in the frequency range of $75-110 \mathrm{GHz}$ has been designed, constructed \& tested for SINP-Tokamak to diagnose second harmonic X- mode electron cyclotron emission (ECE). The selected central frequencies for the 12 channels are given by $74.5,77.5,80.5,83.5,86.5,89.5$, and 94.5, 97.5, 100.5, 103.5, 106.5, 109.5 GHz. The frequency range covers approximately $80 \%$ of the plasma cross-section in case of a magnetic field $\mathrm{B}=1.5 \mathrm{~T}$. For the maximum field of the SINP-Tokamak $\mathrm{B}=2.0 \mathrm{~T}$, about $50 \%$ is covered. The minimum magnetic field is $\mathrm{B}=1.1 \mathrm{~T}$, where only one channel has a corresponding electron cyclotron resonance layer inside the plasma. It is found that harmonic overlap between $2^{\text {nd }}$ and $3^{\text {rd }}$ harmonic occurs at the inside board of the vessel $(22.5$ $<\mathrm{R}<24.0 \mathrm{~cm}$ for $\mathrm{B}=1.5 \mathrm{~T}$ ) as is evident from Fig. 2. The ratio of third and second harmonic cyclotron frequency is always less than $0.1 \%$. The selected bandwidth of each channel is $1 \mathrm{GHz}$, which yields a spatial resolution of $\Delta R / R=1.4 \%$ at the low field side and $0.9 \%$ at the high field side, since the spatial resolution is given by

$$
\frac{\Delta R}{R}=\frac{B_{i f}}{f_{i}}
$$

where $f_{i}$ is the central frequency of a channel. 


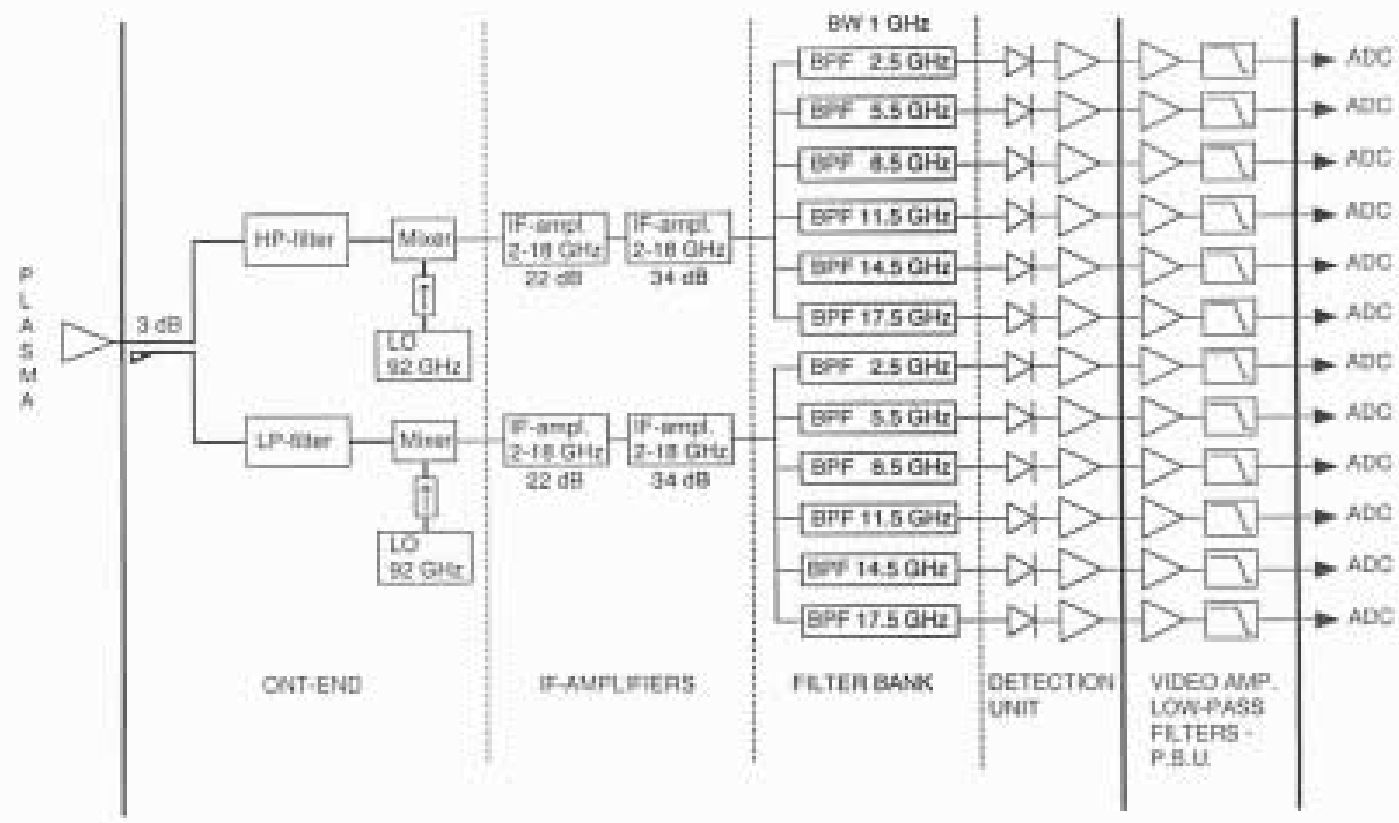

Figure 1. Schematic drawing of the radiometer.
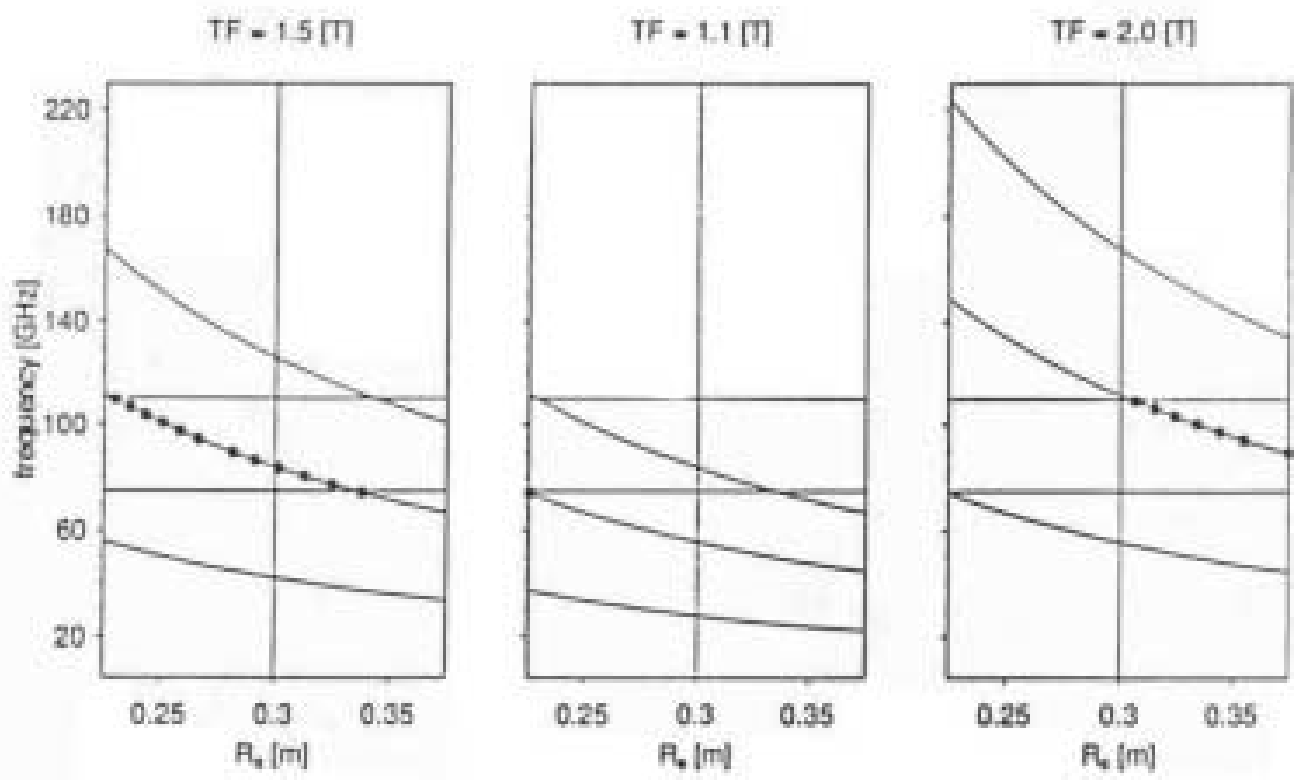

Figure 2. Harmonic overlap between $2^{\text {nd }}$ and $4^{\text {nd }}$ harmonic at the inside board for $\mathrm{B}=1.5 \mathrm{~T}$. 
The following functional requirements were chosen for the SINP-Tokamak.

(a) Frequency range of 75-110 GHz distributed over 12 channels with centre frequencies of 74.5 etc.

(b) The temperature resolution

$$
\frac{\Delta T_{\mathrm{rad}}}{T} \leq 2.5 \%
$$

and

(c) The spatial resolution

$$
\frac{\Delta R}{R}=\frac{B_{i f}}{f_{i}} \leq 2 \%
$$

The radiated power from the plasma is given by

$$
P=k T_{e} B_{i f}
$$

where $P$ is radiated power, $k$ is the Boltzmann constant, $T_{e}$ is plasma temperature and $B_{i f}$ is bandwidth of IF amplifier.

The calculated value of radiated power for $1 \mathrm{GHz}$ bandwidth is found to be $32 \mathrm{nW}$ or $-45 \mathrm{dBm}$.

The noise equivalent temperature can be determined with the help of the following equation:

$$
T_{\text {noise }}=(F-1) T_{\mathrm{amb}}
$$

where $F$ is the overall noise figure of the radiometer and $T_{\mathrm{amb}}$ is the ambient temperature of approximately $300 \mathrm{~K}(25 \mathrm{meV})$. The noise figure $\mathrm{F}$ is defined as the ratio of the signal- to-noise ratio at the input to the signal-to-noise ratio at the output and can be calculated according to:

$$
F=F_{1}+\frac{F_{2}-1}{G_{1}}+\frac{F_{3}-1}{G_{1} G_{2}}+\ldots
$$

where $F_{i}$ and $G_{i}$ are the noise figure and power gain of the individual components respectively.

The ECE radiation is received by a single horn antenna with a gain of approximately $30 \mathrm{~dB}$. The incoming radiation power is split into two branches by a $3 \mathrm{~dB}$ coupler. Before down conversion of the input spectrum, the upper and the lower side band are selected respectively by means of a high pass and a low pass filter. Behind the filters, identical components are employed. The local oscillator frequency is $92 \mathrm{GHz}$ for both mixers. After down conversion the IF frequency range becomes 2 to $18 \mathrm{GHz}$. The IF signal is amplified by two cascaded amplifiers with a total gain of approximately $60 \mathrm{~dB}$. The output of these amplifiers is split into 6 branches. After frequency selection by means of band pass filters, the received power is detected by Schottky diode detectors. After video amplification and filtering the data is recorded by an $\mathrm{ADC}$ with $1 \mathrm{MHz}$ sampling rate. Video low pass filters having cut-off at $300 \mathrm{kHz}$ is used to avoid aliasing. The schematic drawing of the radiometer is shown in Fig. 1.

\section{Calibration \& testing of the radiometer}

Several interesting methods are available for the calibration of ECE radiometer [11]. The SINP radiometer has been tested and calibrated with a high temperature blackbody radiator. The radiation was chopped by a $100 \mathrm{~Hz}$ chopper. The output signal of the radiometer was averaged by means of a digital oscilloscope, with a maximum integration time of 1000 sweeps. The effective input noise temperature for the radiometer channels was found to be in the order of $0.1 \mathrm{eV}$. The linear regime of operation extends up to an input power which corresponds to an electron temperature of approximately $200 \mathrm{eV}$. Above this temperature the $1 \mathrm{~dB}$ compression point of the Schottky diode detectors is exceeded and hence the IF gain needs be appropriately reduced. This can be easily done by incorporating an attenuator behind the IF amplifiers.

The calibration of the SAHA radiometer was performed with a high temperature calibration source to provide thermal emission at $T_{\text {hot }}$. The operating temperature of this source can be set to $600^{\circ} \mathrm{C}$ maximum. The source is placed in front of the receiving antenna of the radiometer. The distance between the source and the radiometer is approximately $40 \mathrm{~cm}$, which is large enough to be in the Fraunhofer region of the antenna, but small enough to cover the complete cross-section of the antenna pattern by the emitting area of the source. A mechanical chopper with blades large enough to block the emission from the source completely is placed between the antenna and the source. The chopper enables the radiometer to detect the $\mathrm{T}_{\text {hot }}$ and the room temperature $\left(T_{\text {cold }}\right)$ thermal emissions alternately. Both the hot source and the chopper blades are placed with a small angle with respect to the position perpendicular to the line of sight of the radiometer to prevent standing waves to influence the measurements. The output waveform of the radiometer is recorded with a digital oscilloscope.

Since the calibration was done at a relatively low radiation temperature compared to the ECE radiation temperature in a tokamak the linearity of the radiometer was checked with the help of a Backward Wave Oscillator (BWO).

\section{Results \& Conclusion}

The combination of a high spatial and temporal resolution, a high sensitivity and an equivalent input noise temperature of a few $\mathrm{eV}$ make the radiometer for the SINP Tokamak a very useful diagnostic. The radiometer is in regular use for the measurement of plasma temperature for toroidal magnetic fields ranging from 1.5 Tesla to nearly 2 Tesla. Efforts have been made to find 
out the temperature profile. A typical temperature radial profile is shown in Fig. 3.

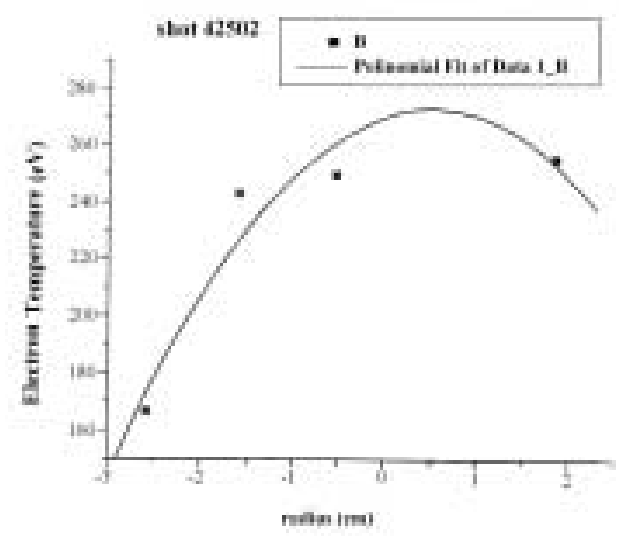

Figure 3. A typical temperature radial profile of the SINP-Tokamak.

\section{Acknowledgement}

The authors are thankful to J. F. M. van Gelder for his contribution in the primary design of the radiometer. The first author sincerely acknowledges the great help received from the FOM Institute of Plasma Physics in The Netherlands for the fabrication and testing of the SINP Radiometer. He gratefully acknowledges the contributions from Dr. (Mrs.) M. S. Janaki of Plasma Physics Division of SINP in the revision of the manuscript. He expresses his thanks to the members of the ECE group of the SINP Tokamak. The authors are thankful to Tony Donne of FOM Institute for his kind assistance in several stages of completion of the radiometer and also for his valuable comments and suggestions.

\section{References}

[1] R.H. Dicke, Rev. Sc. Instrum. 17, 268 (1946).

[2] L.N. Ridenour (Editor-in-Chief), Radiation Laboratory Series, Vol. 1-28, McGraw-Hill, New York (1948).

[3] W.S. Cheung, F.H. Levien, Artech House inc., Norwood (1985).

[4] J.F.M. van Gelder, Thesis: Electron Cyclotron Wave Absorption Experiments in Tokamak Plasmas, The FOM Institute of Plasma Physics, The Netherlands (1996).

[5] G. Bekefi, Radiation Processes in Plasma, Wiley, New York, (1966).

[6] A.E. Costley, R.J. Hastie, J.W.M. Paul and J. Chamberlain, Phys. Rev. Lett. 33, 758 (1974).

[7] M. Bornatici, R. Cano, O. De Barbieri and F. Engelmann, Nucl. Fusion 23, 1153 (1983).

[8] H.J. Hartfuss, T. Geist and M. Hirsch, Plasma Phys. Control. Fusion 39, 1693 (1997).

[9] Santwana Raychaudhuri and S. N. Sengupta, Plasma Phys. \& Controlled Fusion, 34, 475 (1992).

[10] R.P. da Silva, A.M.M. Fonseca, E.R. Calderon and R.M.O. Galvão, Proceeding of the 14th IAEA TCM on Research Using Small Fusion Devices, Sao Paulo, Brazil, June 25-27, (2001).

[11] Kazuo Kawahata, Mizuki Sakamoto, Junji Fujita, Hiroshi Matsuo and Kiyomi Sakai, Japanese Journal of Applied Physics, 29, 1824 (1990). 